\title{
In vivo and in vitro effects of RAD001 on bladder cancer ${ }^{\text {th }}$
}

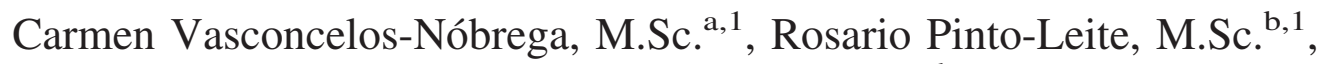 \\ Regina Arantes-Rodrigues, M.Sc. ${ }^{\text {, }}$, Rita Ferreira, Ph.D. ${ }^{\text {, }}$, Paulo Brochado, M.Sc. ,

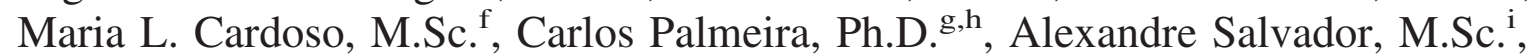 \\ Catarina I. Guedes-Teixeira, M.Sc. ${ }^{c}$, Aura Colaço, Ph.D. ${ }^{c}$, Luis F. Palomino, Ph.D. ${ }^{j}$, \\ Carlos Lopes, Ph.D. ${ }^{k}$, Lúcio Santos, Ph.D. ${ }^{\text {g,h }}$, Paula A. Oliveira, Ph.D. ${ }^{\text {c,* }}$ \\ ${ }^{a}$ Agrarian School of Viseu, Polytechnic Institute of Viseu, Viseu, Portugal \\ ${ }^{\mathrm{b}}$ Genetic Service, Cytogenetic Laboratory, Hospital Center of Trás-os-Montes and Alto Douro, Vila Real, Portugal \\ ${ }^{\mathrm{c}}$ Department of Veterinary Sciences, CECAV, University of Trás-os-Montes and Alto Douro, Vila Real, Portugal \\ ${ }^{\mathrm{d}}$ QOPNA, Mass Spectrometry Center, Department of Chemistry, University of Aveiro, Aveiro, Portugal \\ e Anatomic Pathology Service, Santo António Hospital, Porto, Portugal \\ ${ }^{\mathrm{f}}$ Department of Biochemistry, Faculty of Pharmacology, Oporto University, Porto, Portugal \\ ${ }^{\mathrm{g}}$ Department of Surgical Oncology, Portuguese Institute of Oncology, Porto, Portugal \\ ${ }^{\text {h }}$ Health Faculty, Fernando Pessoa University, Porto, Portugal \\ ${ }^{\mathrm{i}}$ Higher School of Health Technology, Lisbon University, Lisbon, Portugal \\ ${ }^{\mathrm{j}}$ Department of Physiology, Veterinary Faculty, University of Santiago de Compostela, Lugo, Spain \\ ${ }^{\mathrm{k}}$ Abel Salazar Biomedical Sciences Institute-Oporto University (ICBAS-UP), Pathology and Molecular Immunology, Porto, Portugal
}

Received 18 July 2011; received in revised form 24 October 2011; accepted 2 November 2011

\begin{abstract}
Objective: To evaluate the influence of Everolimus (RAD001) on chemically induced urothelial lesions in mice and its influence on in vitro human bladder cancer cell lines.

Methods: ICR male mice were given $N$-butyl- $N$-(4-hydroxybutyl) nitrosamine in drinking water for a period of 12 weeks. Subsequently, RAD001 was administered via oral gavage, for 6 weeks. At the end of the experiment, all the animals were sacrificed and tumor development was determined by means of histopathologic evaluation; mammalian target of rapamycin (mTOR) expressivity was evaluated by immunohistochemistry. Three human bladder cancer cell lines (T24, HT1376, and 5637) were treated using a range of RAD001 concentrations. MTT assay, terminal deoxynucleotidyl transferase dUTP nick end labeling (TUNEL), and flow cytometry were used to assess cell proliferation, apoptosis index, and cell cycle analysis, respectively. Immunoblotting analysis of 3 cell line extracts using mTOR and Akt antibodies was performed in order to study the expression of Akt and mTOR proteins and their phosphorylated forms.

Results: The incidence of urothelial lesions in animals treated with RAD001 was similar to those animals not treated. RAD001 did not block T24 and HT1376 cell proliferation or induce apoptosis. A reduction in cell proliferation rate and therefore $G_{0} / G_{1}$ phase arrest, as well as a statistically significant induction of apoptosis $(P=0.001)$, was only observed in the 5637 cell line.

Conclusion: RAD001 seems not to have a significant effect on chemically induced murine bladder tumors. The effect of RAD001 on tumor proliferation and apoptosis was achieved only in superficial derived bladder cancer cell line, no effect was observed in invasive cell lines. (c) 2011 Elsevier Inc. All rights reserved.
\end{abstract}

Keywords: RAD001; Mice; $N$-butyl- $N$-(4-hydroxybutyl) nitrosamine; Bladder cancer cell lines; Proliferation; Apoptosis; mTOR; Akt expressivity

\section{Introduction}

Cancer is a major public health problem worldwide.

This study was supported by an aid grant from the Fundação para a Ciência e Tecnologia, Ministério da Ciência e Ensino Superior, Portugal, grant number SFRH/BD/40896/2007.

* Corresponding author. Tel.: +00351966473062; fax: +351259350480.

E-mail address: pamo@utad.pt (P.A. Oliveira).

${ }^{1}$ These authors contributed equally to this work.
Bladder cancer is the second most common tumor of the urinary tract $[1,2]$ and its incidence over the last half century has increased. Urothelial tumors of the bladder are usually superficial at presentation in $70 \%$ to $80 \%$ of patients, and invasive in $20 \%$ to $30 \%$ [3]. The invasive and metastatic 


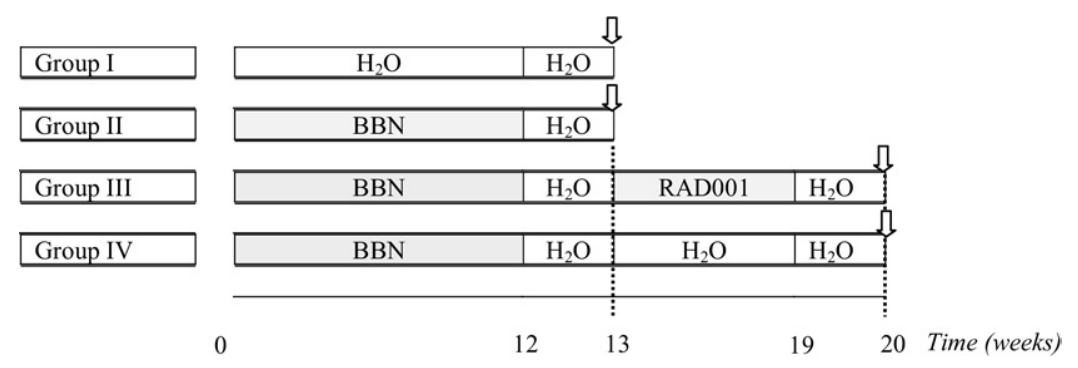

Fig. 1. Experimental design (arrows represent time period after which euthanasia took place).

bladder cancers represents the leading cause of morbidity and mortality among bladder cancer patients because the therapeutic protocols currently available fail to provide an effective response or to provide positive outcomes. New strategies and new therapeutic agents should therefore be tested.

Animal models of bladder cancer have been widely used in the preclinical development of new and more effective therapeutic agents, as well as to enable a deeper and more accurate understanding of the process of bladder carcinogenesis [4,5]. Oral administration over a period of 12 weeks [6] of $N$-butyl- $N$-(4-hydroxybutyl) nitrosamine (BBN) induces the development of invasive urothelial cancers that exhibit similar morphological characteristics and molecular events, such as their DNA content, proliferative index, and p53 overexpression, as human urothelial bladder cancer [7].

Activation of the PI3K/Akt/mTOR axis was associated with early events in carcinogenesis and the interruption of pathways, which led to antiproliferation, antisurvival, antiangiogenic, and proapoptotic effects [8].

RAD001 (everolimus) is an oral mTOR inhibitor with an antineoplastic activity similar to rapamycin (sirolimus), an anticancer agent used on solid tumors [9-13]. RAD001 has a potent antiproliferative effect when used against human breast cancer cell lines, lymphoma cells, prostate cancer cells, and gastric cancer [14]. Its therapeutic effects are under clinical evaluation for use on different types of solid cancer, including renal cell and endometrial cancer [15]. It has a shorter half-life and greater bioavailability than rapamycin and after entering cells it binds with FKBP-12, an immunophilin. This forms a complex that binds to the mammalian target of rapamycin (TOR), thus inhibiting growth factor induced transduction signals that mediate cellular division, retarding, or arresting the cell cycle in $G_{1}$ phase or, in some cases, inducing apoptosis [12,14,16-19].

In this study, we evaluated the therapeutic effects of RAD001 on mice with BBN induced neoplastic urothelial lesions similar to those found in humans, as well as its effect in vitro on 3 human bladder cancer cell lines (T24, HT1376, and 5637).

\section{Materials and methods}

\subsection{Chemicals}

BBN was purchased from Tokyo Kasei Kogyo Co. Ltd. (Tokyo, Japan), RAD001 (Certican) was purchased from Novartis (Basel, Switzerland) (for in vivo study) and from Sigma (St. Louis, MO) (for in vitro study).

\subsection{In vivo studies}

\subsubsection{Animals}

Four week old male ICR mice were obtained from Harlan Interfauna Inc. and housed in plastic cages with wood chips for bedding. All mice used in the experiment were acclimatized for 1 week under routine laboratory conditions before starting the experiments. They were placed in a temperature $\left(23 \pm 2^{\circ} \mathrm{C}\right)$ and humidity $(50 \% \pm 10 \%)$ controlled environment in which a 12:12 hour light:dark cycle was maintained. All animals had free access to water and a standard lab diet, and all procedures were performed in accordance with the European Communities Council Directive $86 / 609 /$ ECC.

\subsubsection{Animal experiments}

After 1 week of quarantine, a total of 51 ICR mice were randomly divided into 4 groups (Fig. 1) (group I: $n=10$; group II: $n=12$; group III: $n=15$; group IV: $n=14$ ), groups II, III, and IV received BBN $(0.05 \%)$ in drinking water for 12 weeks. Group I was used as a negative control group, drinking only tap water. One week after bringing BBN exposure to an end, groups I and II were euthanized by means of pentobarbital overdose anesthesia to evaluate the presence of any preneoplastic and neoplasic lesions induced by BBN. After being treated with BBN solution for 12 weeks, groups III and IV were maintained with normal tap water during 1 additional week. Group III was treated with RAD001, 2 days a week (5 mg/kg) [20] by oral gavage for 6 consecutive weeks, until the 19th week of the experiment, with group IV being used as the treatment control group.

For the duration of the study, the mice's state of health was monitored daily. The animals' drinking solution was changed once a week or earlier if necessary, and the volume 
drunk was recorded. Weekly food intake was also noted. Their body weights were initially measured once a week and then twice a week after treatment had begun, allowing us to adjust RAD001 doses to individual weight variations.

\subsubsection{Evaluation of treatment}

All surviving animals from groups III and IV were euthanized, according to the technique described above, 1 week after ending RAD001 treatment. Necropsies were carried out and urinary bladders of the mice were collected according to the technique described by Oliveira et al. (2009) [6]. After overnight fixation, bladders were cut longitudinally and their mucosal surface was carefully examined for the existence of macroscopic lesions. After this procedure, they were embedded in paraffin and $2 \mu \mathrm{m}$ sections were cut and stained with hematoxylin and eosin. All other organs were also examined macroscopically for any changes, and their weights were recorded.

\subsection{Histology}

Histologic slides were observed under a light microscope by 2 investigators. All slides were examined without prior knowledge of the treatment given to the animals whose tissue samples were under investigation. Histologic lesions found in different groups were classified and staged according to World Health Organization guidelines [21]. Urothelial lesions were categorized as simple hyperplasia, nodular hyperplasia, dysplasia, carcinoma in situ, papilloma, low grade non invasive papillary urothelial carcinoma, high grade non invasive papillary urothelial carcinoma, invasive urothelial carcinoma, and squamous metaplasia.

\subsection{Immunohistochemistry}

The mTOR (7C10) rabbit mAb (\#2983; Cell Signaling Technology, Danvers, MA) was used as the primary antibody. The immunohistochemistry assay was performed with an autostainer (LVI; Labvision Corporation, Cheshire, UK) using the Ultra VisionHP-125-HL detection kit (Labvision Corporation). The staining procedure using this kit is based on the labeled streptavidin biotin method. Heat antigen retrieval was performed by slides pretreatment in a jar with sodium citrate buffer $\mathrm{pH} 6$ and heated 3 times for 5 minutes in a microwave oven at $700 \mathrm{~W}$. Slides were incubated 60 minutes at $37^{\circ} \mathrm{C}$ with the primary antibody (1:50). Paraffin sections from human lung carcinoma were used as a positive control.

\subsection{Immunohistochemical evaluation}

Sections were examined under light microscopy. The immunohistochemical expression was classified as exhibiting positive staining if the pattern of immunoreactivity was identical to that of the cytoplasm staining of normal urothe- lial cells for mTOR activity. Negative staining was considered if lesions/tumors showed no immunoreactivity.

\subsection{In vitro studies}

\subsubsection{Tumor cell lines and culture conditions}

In vitro study was performed using 2 established human invasive bladder cancer cell lines: T24 and HT1376, and 1 superficial urothelial bladder cancer cell line: 5637 (the first line was provided by DSMZ, Düsseldorf, Germany and the last 2 lines provided by Dr. Paula Videira of the Universidade Nova de Lisboa). All the cell lines were cultured as a monolayer in RPMI 1640 culture medium (PAA, Pasching, Austria) supplemented with $10 \%$ heat inactivated fetal calf serum (Biological Industries, Kibbutz Beit Haemek, Israel), $100 \mathrm{U} / \mathrm{mL}$ penicillin (Biological Industries), $100 \mu \mathrm{g} / \mathrm{mL}$ streptomycin (Biological Industries) and $2 \mathrm{mM}$ L-Glutamine (Sigma Aldrich, St. Louis, MO). Cells were maintained at $37^{\circ} \mathrm{C}$ in a humidified atmosphere with $5 \% \mathrm{CO}_{2}-$ $95 \% \mathrm{O}_{2}$ and were routinely subcultured by trypsination. All experiments were performed during exponential cell growth. All bladder cancer cell lines expressed higher amounts of VEGF-A, however, the 5637 cell line had the highest VEGF-A expression of any cell line studied [22].

\subsubsection{Drug exposure}

Bladder cancer cell lines were exposed to concentrations of RAD001 ranging from 0 to $2 \mu \mathrm{M}(0 ; 0.05 ; 0.1 ; 1 ; 2 \mu \mathrm{M})$ over the course of 72 hours to assess dose response profiles. An untreated control group was processed in the same way as the treatment samples but in a drug free medium, and the evaluation of the cytotoxicity effect was performed immediately after the end of drug exposure. The concentration of $\mathrm{RAD} 001$ represented the average $\mathrm{IC}_{30}$ dose at 72 hours from each respective single concentration. $\mathrm{IC}_{30}$ is defined as the drug concentration that causes a $30 \%$ reduction in cell numbers compared with that of the untreated control $\left(\mathrm{IC}_{30}=30 \%\right.$ growth inhibition $)$ [23].

\subsubsection{Cell viability assay}

The MTT (3-[4,5-dimethylthiazol-2-yl]-2,5-diphenyl tetrazolium bromide; Sigma Aldrich) assay was used to assess the relative percentage of metabolically active cells relative to untreated controls [24]. In brief, trypsinized tumor cells were resuspended in a medium at $2-3 \times 10^{4}$ cells $/ \mathrm{ml}$, based on the growth characteristics of each cell line after verifying cell viability via trypan blue dye exclusion. One hundred $\mu 1$ of cell suspension were seeded into each well of a 96 well flat bottom microtiter (Sarstedt, Newton, NC) and plates were incubated for 24 hours to allow adherent cell growth. After overnight incubation, the medium was removed and $100 \mu \mathrm{L}$ of the different reagent solutions in complete medium and medium control were distributed in each well and incubated for 72 hours. After incubation, $10 \mu \mathrm{L}$ of MTT dye working solution $(5 \mathrm{mg} / \mathrm{ml})$ was added to each well. After 4 hours of incubation, the supernatant in the wells was re- 
Table 1

Mean and relative weight of bladder, kidney, and liver (value \pm SD)

\begin{tabular}{|c|c|c|c|c|c|c|c|c|}
\hline \multirow[t]{2}{*}{ Group } & \multicolumn{4}{|l|}{ Mean weight (g) } & \multicolumn{4}{|l|}{ Relative weight (g) } \\
\hline & Bladder & Right kidney & Left kidney & Liver & Bladder & Right kidney & Left kidney & Liver \\
\hline I & $0.280 \pm 0.113$ & $0.327 \pm 0.018$ & $0.341 \pm 0.042$ & $2.32 \pm 0.41^{\mathrm{c}}$ & $0.063 \pm 0.0022$ & $0.0075 \pm 0.00054^{\mathrm{f}}$ & $0.0078 \pm 0.001^{\mathrm{g}}$ & $0.053 \pm 0.0091$ \\
\hline II & $0.269 \pm 0.115$ & $0.347 \pm 0.041$ & $0.343 \pm 0.051$ & $1.95 \pm 0.28$ & $0.069 \pm 0.003$ & $0.009 \pm 0.001$ & $0.0089 \pm 0.001$ & $0.050 \pm 0.0051$ \\
\hline III & $0.167 \pm 0.05^{\mathrm{a}}$ & $0.328 \pm 0.091$ & $0.292 \pm 0.0107^{\mathrm{b}}$ & $2.30 \pm 0.46^{\mathrm{d}}$ & $0.004 \pm 0.001^{\mathrm{e}}$ & $0.008 \pm 0.002$ & $0.0072 \pm 0.0026$ & $0.0566 \pm 0.0110^{\mathrm{h}}$ \\
\hline IV & $0.38 \pm 0.28$ & $0.376 \pm 0.059$ & $0.407 \pm 0.165$ & $2.08 \pm 0.251$ & $0.0094 \pm 0.0077$ & $0.0089 \pm 0.001$ & $0.0097 \pm 0.0042$ & $0.0495 \pm 0.0047$ \\
\hline
\end{tabular}

$\mathrm{SD}=$ standard deviation.

${ }^{\mathrm{a}}$ Different from group IV $(P=0.01) .{ }^{\mathrm{b}}$ Different from group IV $(P=0.041)$. ${ }^{\mathrm{c}}$ Different from group II $(P=0.0024)$. ${ }^{\mathrm{d}}$ Different from group IV $(P=$ 0.0139). ${ }^{\mathrm{e}}$ Different from group IV $(P=0.017) .{ }^{\mathrm{f}}$ Different from group II $(P=0.003) .{ }^{\mathrm{g}}$ Different from group II $(P=0.045)$. ${ }^{\mathrm{h}}$ Different from group IV $(P=0.033)$.

moved and replaced by $100 \mu \mathrm{l} /$ well of dimethylsulfoxide (DMSO, Sigma Aldrich). The absorbance (A) values of each well were recorded at $492 \mathrm{~nm}$ on an automatic ELISA plate reader (Multiskan EX; Labsystems). The medium containing tetrazolium dye but without cells (blank), was used as a negative control. After the blank correction, the relative viability was calculated as follows: $A_{\text {exp group }} / A_{\text {control }} \times 100$ $[20,25]$. In each experiment, triplicate wells were performed for each RAD001 concentration and the assay was repeated in 2 independent experiment replications.

\subsubsection{TUNEL assay}

In order to detect and quantify DNA strand breaks in apoptotic bladder cells, terminal deoxynucleotidyl transferase dUTP nick end labeling (TUNEL) assay was performed. Cells $\left(2 \times 10^{4}\right)$ were then seeded and allowed to attach overnight. Subsequently, cells were treated with RAD001 $(2 \mu \mathrm{M})$ and incubated for 72 hours. After treatment, cells were trypsinized, washed with PBS, and fixed with $4 \%$ paraformaldehyde for 1 hour at 15 to $25^{\circ} \mathrm{C}$. The in situ cell death detection kit fluorescein (Roche, IN) was adapted from the manufacturer's instructions. Slides were observed using a fluorescence microscope (Nikon Eclipse E400, Tokyo, Japan). Cells were defined as apoptotic if the nuclear area of cells was labeled positively (green color). The number of cells undergoing apoptosis was counted in random fields on each slide. At least 200 cells were counted (magnification $1000 \times$ ) in random fields on each slide. The apoptotic index (AI) was determined as follows: $\mathrm{AI}(\%)=$ (number of apoptotic cells/total number of cells) $\times 100$ [26].

\subsubsection{Cell cycle analysis}

Cells $\left(1 \times 10^{6}\right)$ were plated in 6 well plates and incubated for 24 hours. Then RAD001 was added in serial dilutions. After 72 hours of incubation, cells were trypsinized, washed with PBS, and fixed in ice cold ethanol $70 \%$ for at least 1 hour. Propidium iodide (PI, Cycloscope DNA Cytometry, Cytognos, Maia, Portugal) was added in order to label total cellular DNA, and cell cycle analysis was carried out using a Coulter EPICS XL-MCL flow cytometer. DNA-content histograms were analyzed with Modfit LT 3.0 software (Verity) to determine the percentage of cells in each cell cycle: $\mathrm{G}_{0} / \mathrm{G}_{1}, \mathrm{~S}$ and $\mathrm{G}_{2} / \mathrm{M}$. Cells with DNA content less than that of $\mathrm{G}_{0} / \mathrm{G}_{1}$-phase cells were considered to be apoptotic (sub- $\mathrm{G}_{0} / \mathrm{G}_{1}$ ). Each independent experiment was performed in triplicate.

\section{7. mTOR and Akt expression analysis}

Whole cell extracts were obtained from mechanical homogenization of cell pellets in $1 \%$ SDS containing phos-

Table 2

Incidence of urothelial lesions in ICR mice exposed to $N$-butyl- $N$-(4-hydroxybutyl) nitrosamine and treated with RAD001

\begin{tabular}{|c|c|c|c|c|}
\hline Histologic lesion & $\begin{array}{l}\text { Group } \mathrm{I} \mathrm{H}_{2} \mathrm{O} \\
(n=10)\end{array}$ & $\begin{array}{l}\text { Group II BBN } \\
(n=12)\end{array}$ & $\begin{array}{l}\text { Group III BBN+RAD001 } \\
(n=14)\end{array}$ & $\begin{array}{l}\text { Group IV } \mathrm{BBN}+\mathrm{H}_{2} \mathrm{O} \\
(n=13)\end{array}$ \\
\hline Normal urothelium & $10(100 \%)$ & $0(0 \%)$ & $0(0 \%)$ & $0(0 \%)$ \\
\hline Simple hyperplasia & $0(0 \%)$ & $8(67 \%)$ & $9(64.3 \%)$ & $10(76.9 \%)$ \\
\hline Nodular hyperplasia & $0(0 \%)$ & $7(58.3 \%)$ & $8(57.14 \%)$ & $9(69.23 \%)$ \\
\hline Dysplasia & $0(0 \%)$ & $12(100 \%)$ & $12(85.7 \%)$ & $13(100 \%)$ \\
\hline Carcinoma in situ & $0(0 \%)$ & $4(33 \%)$ & $1(7.1 \%)$ & $2(15.38 \%)$ \\
\hline Papilloma & $0(0 \%)$ & $0(0 \%)$ & $1(7.1 \%)$ & $0(0 \%)$ \\
\hline $\begin{array}{l}\text { Low-grade noninvasive papillary } \\
\text { urothelial carcinoma }\end{array}$ & $0(0 \%)$ & $0(0 \%)$ & $3(21.4 \%)$ & $1(7.69 \%)$ \\
\hline $\begin{array}{l}\text { High-grade noninvasive papillary } \\
\text { urothelial carcinoma }\end{array}$ & $0(0 \%)$ & $0(0 \%)$ & $0(0 \%)$ & $1(7.69 \%)$ \\
\hline Invasive urothelial carcinoma & $0(0 \%)$ & $4(33 \%)$ & $6(42.86 \%)$ & $7(53.84 \%)$ \\
\hline Squamous metaplasia & $0(0 \%)$ & $8(67 \%)$ & $9(64.3 \%)$ & $10(76.9 \%)$ \\
\hline
\end{tabular}


phatase inhibitors (P0044 and P5726, Sigma). Protein concentration was assayed by the colorimetric method "RC DC protein assay" (Bio-Rad, Foster City, CA) using bovine serum albumin (BSA) as standard. Samples from each cell line, with no treatment and with $2 \mu \mathrm{M}$ RAD001, were then diluted in Tris buffered saline (TBS; $100 \mathrm{mM}$ Tris, $1.5 \mathrm{mM}$ $\mathrm{NaCl}, \mathrm{pH} 8.0$ ) to obtain a final protein concentration of 0.4 $\mathrm{mg} / \mathrm{mL}$ and a volume of $100 \mu \mathrm{l}$ was slot-blotted into a nitrocellulose membrane (Whatman, Protan, Kent, UK). Nonspecific binding was blocked with 5\% (wt/vol) BSA in TBS-T (TBS with 0.5\% Tween 20) for 1 hour, and the membrane was then incubated with primary antibody (1: 1000 dilution; rabbit anti-mTOR, \#2983, rabbit anti-Phospho-mTOR, \#2971, rabbit anti-Akt, \#9272, or rabbit antiPhospho-Akt, \#4058 from Cell Signaling, St. Louis, MO). After 1 hour incubation, the membrane was washed with TBS-T and incubated with anti-rabbit IgG peroxidase secondary antibody (1:1000; Amersham Pharmacia Biotech). Immunoreactivity was detected with enhanced chemiluminescence reagents (ECL, Amersham Pharmacia Biotech, Piscataway, NJ) according to the manufacturer's instructions, and images were recorded using X-ray films (Kodak Biomax Light Film, Sigma). Films were scanned in Molecular Imager Gel Doc XR + System (Bio-Rad) and analyzed with QuantityOne software ver. 4.6.3 (Bio-Rad). Protein loading was controlled with Ponceau S staining.

\subsubsection{Statistical analysis}

Statistical analysis was carried out using the SPSS 17.0 statistical software (SPSS Inc., Chicago, IL). Body weight differences and the mean and relative weights of the animals' livers, kidneys and bladders were assessed using ANOVA, with Bonferroni multiple comparison. Nonparametric data were computed using the $\chi^{2}$ test or Fisher's exact test with Bonferroni's correction. For in vitro studies, ANOVA was performed, the equality of variances was tested by Levene F test, and the statistical significance of differences between the treatment and control groups were determined by Dunnett's Multiple Comparison post-hoc test for the MTT assay. The Pearson product-moment correla- tion coefficient was used to evaluate the correlation (linear dependence) of the cell cycle, apoptosis, and RAD001 concentration. $P$ values $<0.05$ were considered to be statistically significant.

\section{Results}

\subsection{In vivo results}

\subsubsection{Animal growth and water and food consumption}

The mean food and water intake was constant and similar across the different groups throughout the whole study period (data not shown).

Two mice one from each group III and IV died during the experiment and therefore only surviving animals were considered for histopathologic examination. During the treatment period, animals exhibited normal cage activity. Comparing the body weight of mice treated and those not treated with RAD001, the differences were not statistically significant.

The mean weight of the animals' livers, kidneys, and bladders, as well as the relative organ weights (calculated as the ratio of the mouse's organ weight by the mouse's weight) are shown in Table 1.

\subsection{Macroscopic and microscopic evaluation}

Greyish-white urinary bladder masses which varied in size were observed in groups II, III, and IV. The lesions were invasive or less frequently pedunculated. The lesions exhibited irregular surfaces and hemorrhage, while they were distributed randomly throughout the entire urinary bladder. No macroscopic urinary bladder changes were identified in the control group (I). Stone formation in the urinary bladder was not observed in any of the mice. No macroscopic changes were seen in the liver, lung, kidneys, and gastrointestinal tract of the animals involved.

Table 3

Cytoplasm immunoreactivity of urothelial lesions with mTOR antibody in ICR mice exposed to $N$-butyl- $N$-(4-hydroxybutyl) nitrosamine and treated with RAD001

\begin{tabular}{|c|c|c|c|}
\hline Group & Histologic lesion & Positive & Negative \\
\hline \multirow[t]{3}{*}{ Group I $\left(\mathrm{H}_{2} \mathrm{O}\right)$} & Normal urothelium $(n=10)$ & $10(100 \%)$ & - \\
\hline & Simple hyperplasia $(n=9)$ & $9(100 \%)$ & - \\
\hline & Nodular hyperplasia $(n=5)$ & $3(60 \%)$ & $2(40 \%)$ \\
\hline \multirow[t]{5}{*}{ Group III (BBN+RAD001) } & Dysplasia $(n=12)$ & $12(100 \%)$ & - \\
\hline & Invasive urothelial carcinoma $(n=5)$ & $1(20 \%)$ & $4(80 \%)$ \\
\hline & Squamous metaplasia $(n=6)$ & $2(33.3 \%)$ & $4(66.7 \%)$ \\
\hline & Simple hyperplasia $(n=9)$ & $9(100 \%)$ & - \\
\hline & Nodular hyperplasia $(n=3)$ & $3(100 \%)$ & - \\
\hline \multirow[t]{3}{*}{ Group IV $\left(\mathrm{BBN}+\mathrm{H}_{2} \mathrm{O}\right)$} & Dysplasia $(n=8)$ & $8(100 \%)$ & - \\
\hline & Invasive carcinoma $(n=4)$ & $3(75 \%)$ & $1(25 \%)$ \\
\hline & Squamous metaplasia $(n=6)$ & $5(83.3 \%)$ & $1(16.7 \%)$ \\
\hline
\end{tabular}




\subsection{Effects of $B B N$ on urothelial tumorigenesis}

We conducted detailed histopathologic examination to determine the degree of urothelial lesions induced by BBN. These occurred only in the groups drinking BBN solution. Animals from group I (control group) did not show any histologic alterations, i.e., $100 \%$ of them exhibited a normal urothelium. Table 2 summarizes the incidence of BBNinduced urothelial lesions in each group.

\subsection{Effects of RAD001 on urothelial tumorigenesis}

The incidence of lesions between groups treated with RAD001 and those not treated can be observed in Table 2 . Histologic alterations, such as simple and nodular hyperplasia, dysplasia, and carcinoma in situ, exhibited a lower incidence in animals treated with RAD001 (group III) than animals not treated (group IV). There was a higher inci- dence of papillomas and low-grade noninvasive papillary urothelial carcinomas in animals treated with RAD001 compared with animals not treated. The incidence of BBNinduced invasive urothelial carcinoma was slightly lower in mice treated with RAD001 (group III) and these differences were not statistically significant.

\subsection{Immunohistochemistry}

mTOR immunoreactivity was performed in urinary bladders samples from groups I, III, and IV. The results of the mTOR immunoreactivity investigations are summarized in Table 3 and discussed below using the diagnostic categories reported on Table 2. In normal urothelium (Fig. 2A), the cytoplasm was uniformly stained. Simple hyperplasia (Fig. 2B) and dysplasia (Fig. 2C) showed a similar mTOR pattern compared with normal urothelium, with its expression confined to urothelium cytoplasm. On squamous metaplasia

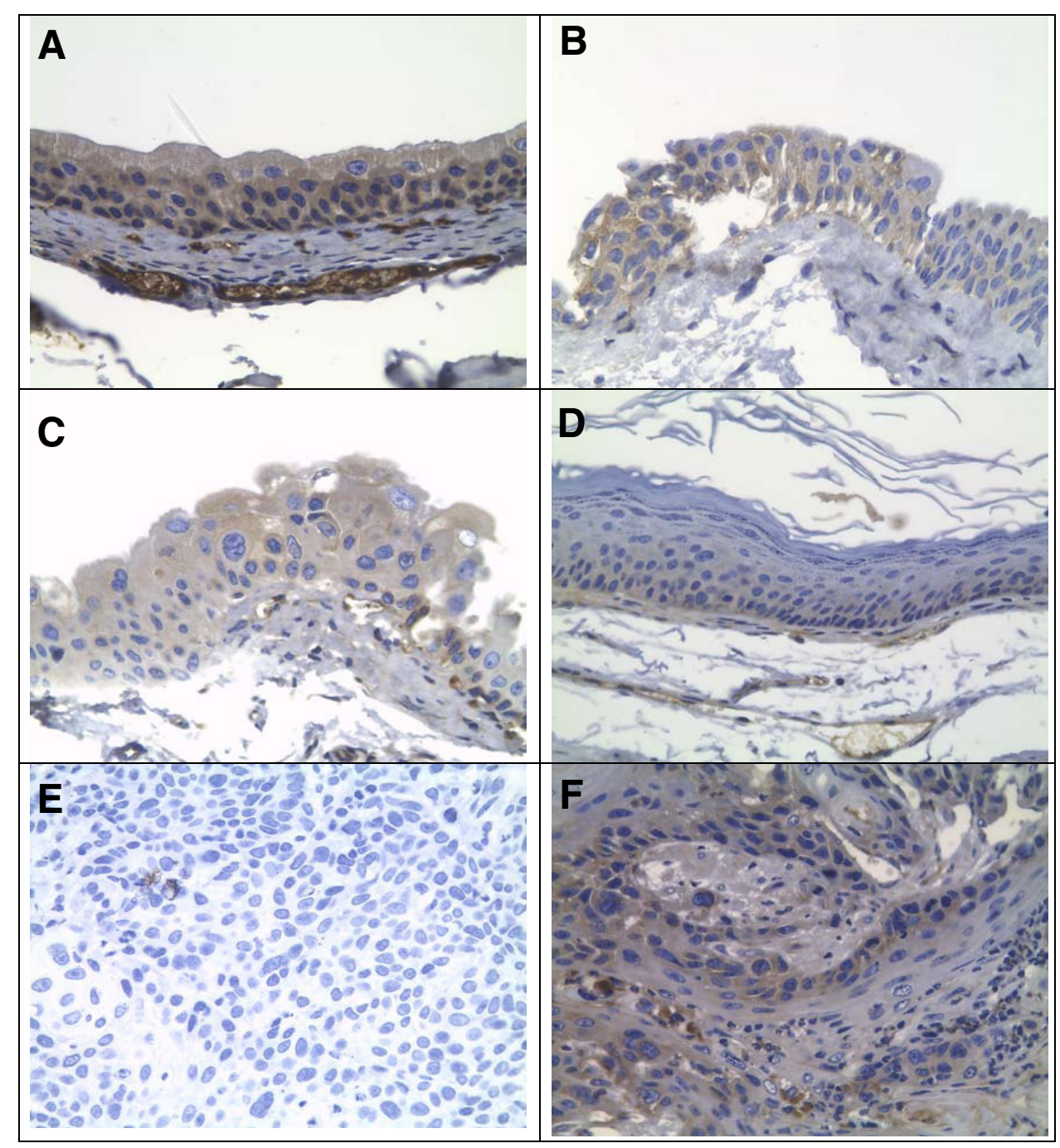

Fig. 2. (A) mTOR expression in normal mouse urothelium (400×); (B) mTOR expression in simple hyperplasia (400×); (C) mTOR expression in dysplasia $(400 \times)$; (D) mTOR expression in squamous metaplasia $(400 \times)$; (E) absence of mTOR expression in invasive carcinoma $(400 \times)$; $(\mathrm{F}) \mathrm{mTOR}$ expression in invasive carcinoma $(400 \times)$. (Color version of figure is available online.) 


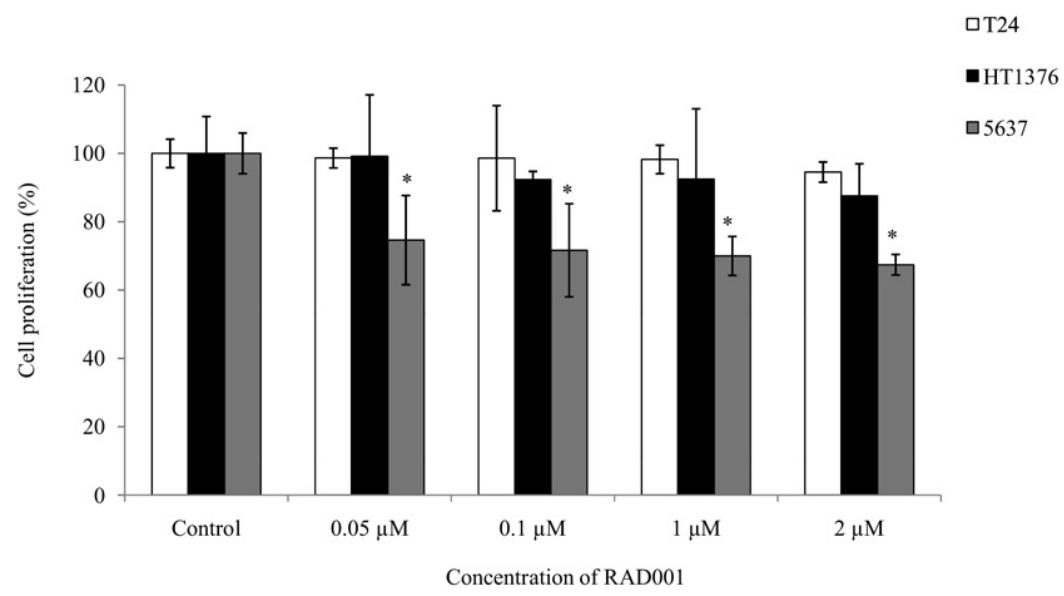

Fig. 3. Bladder cell lines (T24, HT1376 and 5637) were treated with different doses of RAD001 and cell proliferation was monitored using the MTT assay. The data shown and bars represent the mean values $\pm \mathrm{SD}$. $* P<0.05$ versus untreated controls.

(Fig. 2D), we observed a homogeneous cytoplasm immunoreactivity pattern on basal layer, and loss of immunoreactivity in upper layers. mTOR immunohistochemical stain performed on invasive carcinoma from animals treated with RAD001 (Fig. 2E) revealed no staining in $80 \%$ of the lesions observed. However, $75 \%$ of invasive carcinomas in animals not treated revealed positive cytoplasm immunoreactivity (Fig. 2F).

\subsection{In vitro results}

\subsubsection{Cytotoxic effect of RAD001 against bladder cancer cell lines}

RAD001 was added to the 3 bladder cancer cell lines and its proliferation was quantified 72 hours after plating. The cytotoxic activity of RAD001 is shown in Fig. 3. The IC values were determined by MTT assay, according to exposure time and dose of agent. RAD001 transiently inhibited bladder cancer cell growth in a dose-dependent manner. RAD001 is more effective on 5637 cells and exhibits an $\mathrm{IC}_{30}$ at $1 \mu \mathrm{M}$. Among the 3 cell lines tested, T24 cells demonstrated significant resistance to RAD001 exposition, as clearly documented by survival rates of $98.6 \%, 98.5 \%$, $98.2 \%$, and $94.5 \%$, and were the least sensitive to RAD001, compared with HT1376 cell-line survival rates (99.2\%, $92.3 \%, 92.4 \%$, and $87.5 \%$ ). Statistically, the anti-proliferative activity of RAD001 was only significant in the 5637 cell line, at all concentrations, compared with the control group $(P<0.05)$.

Table 4

Apoptotic index values (\%) in the three bladder-cancer cell lines after exposure to RAD001

\begin{tabular}{llll}
\hline Groups & T24 & 5637 & HT1376 \\
\hline Control & $1.5 \%$ & $3 \%$ & $1.5 \%$ \\
RAD001 $(2 \mu \mathrm{M})$ & $2.5 \%$ & $6.5 \%$ & $1.5 \%$ \\
\hline
\end{tabular}

\subsection{TUNEL assay}

To analyze RAD001 induced apoptosis in bladder-cancer cells in vitro, TUNEL was carried out. Only an apoptotic index value of $6.5 \%$ in 5637 cell line was observed as a maximum apoptotic effect in this study. In T24 and HT1376 cell lines, this effect was minimal (Table 4).

\subsection{Cell cycle and apoptosis analysis}

The comparative analysis of the percentage of cells in each cell cycle phase, spanning the control experiment and the different drug concentrations tested, is summarized in Fig. 4. Cell cycle alterations were only observed in the 5637 bladder cancer cell line, namely a decrease in the percentage of cells in S-phase fraction $(P=0.029)$ between the control and treated cells. This effect on proliferative rate was inversely correlated to drug concentration $(\mathrm{r}=-0.978 ; P=$ 0.004).

Regarding the relationship between drug treatment and apoptosis, no effects were observed in invasive bladdercancer cell lines T24 and HT1376 (Fig. 5). Only in the 5637 superficial cell line, a slight but statistically significant increase in sub- $\mathrm{G}_{0} / \mathrm{G}_{1}$ fraction $(P=0.001)$ was detected, an effect which was positively correlated with concentrations of RAD001 $(\mathrm{r}=0.956 ; P=0.011)$.

\subsection{Effects of RAD001 on mTOR and Akt activation}

To analyze RAD001 effect on mTOR activation, the expression of mTOR and its phosphorylated form was analyzed in all the cell lines. As can be depicted in Fig. 6 , no significant expression differences of mTOR and on its phosphorylation at $\mathrm{Ser}^{2448}$ were found due to RAD001 administration in cell lines T24 and HT1376. In cell line 5637, a significant decrease of mTOR expression was noticed, though not paralleled by a significant increase in 

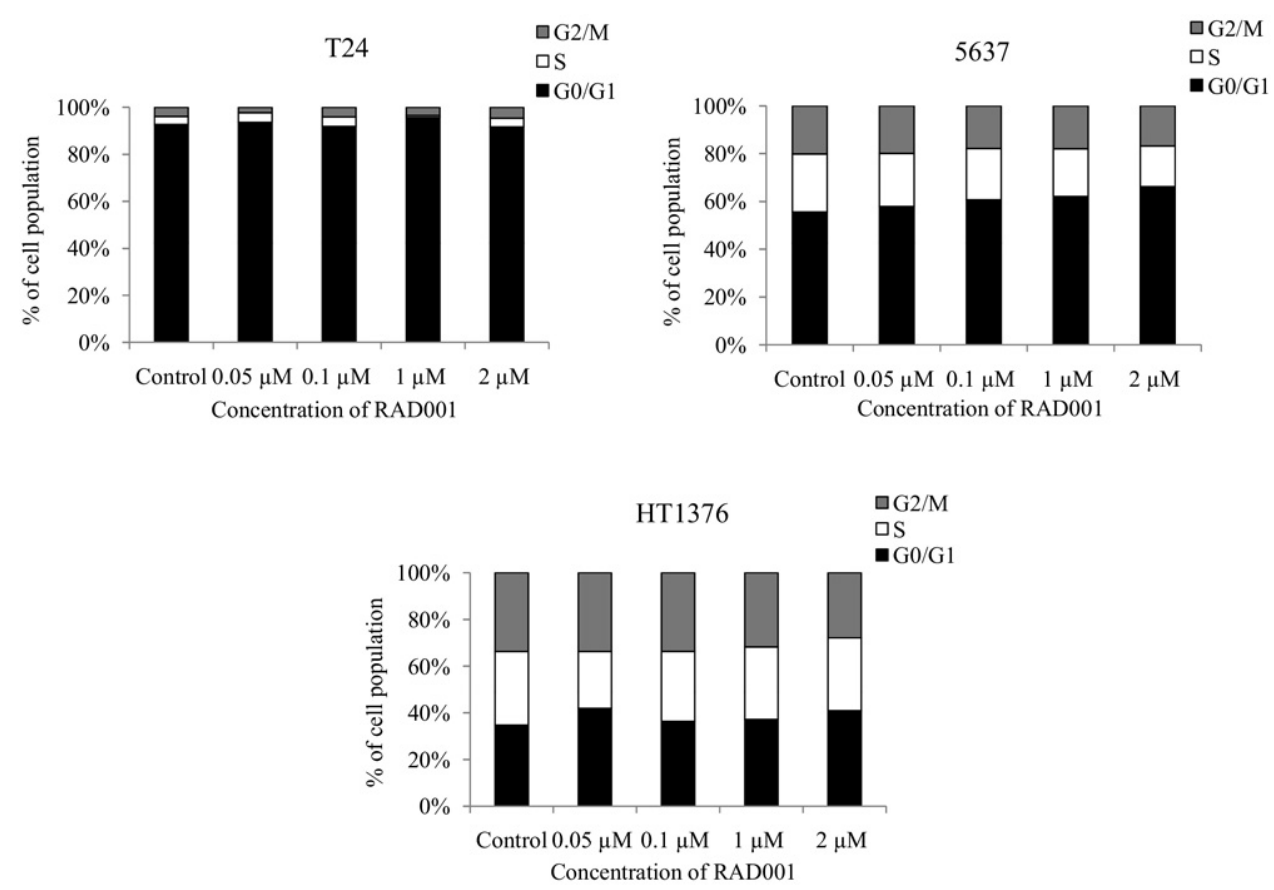

Fig. 4. Cell cycle analysis of the T24, 5637, and HT1376 bladder cancer cell lines after exposure to different concentration of RAD001. The percentages of cells in each cell cycle phases are mean \pm SD of 3 independent experiments. SD $=$ standard deviation.

its phosphorylation. Once the major PI3K pathway led from Akt to mTOR, the expression of Akt was also evaluated by immunodetection of Akt and its phosphorylated form. RAD001 treatment resulted in a slight increase in the baseline expression of Akt in T24 and HT1376 cell lines, and of its phosphorylation at Ser [473] in HT1376 and 5637 cell lines.

\section{Discussion}

The mouse model of invasive bladder cancer induced by $\mathrm{BBN}$, as well as other animal models, plays an essential role

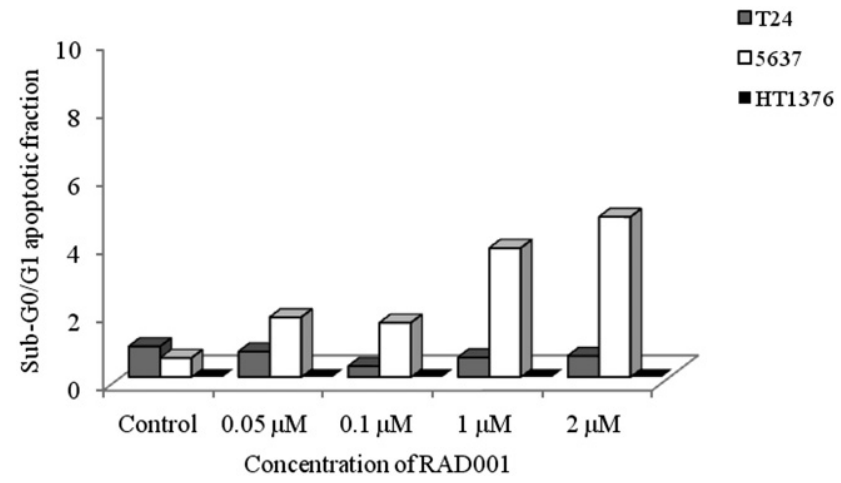

Fig. 5. Sub-G0/G1 fraction on bladder-cancer cell lines T24, 5637, and HT1376, after exposure to different concentration of RAD001. Sub-G0/G1 values are mean $\pm \mathrm{SD}$ of 3 independent experiments. For HT1376, the values were zero for all the experimental conditions. $\mathrm{SD}=$ standard deviation. in the evaluation of target cancer therapy. Disruption of the mTOR pathway has been found in a number of human tumors resulting in intracellular pro-survival and proliferative staining. In this study, we investigated the effect of the mTOR inhibitor RAD001 on BBN induced bladder tumorigenesis in mice and human bladder cancer cells. This investigation took into account our previous studies concerning the effects of sirolimus on the development of urothelial lesions induced by BBN in mice and the in vitro effects on the T24 cancer cell line [24,27]. For this purpose, male ICR mice were exposed to BBN for 12 weeks and later treated with RAD001 twice per week, for 6 consecutive weeks. Three human bladder-cancer cell lines were treated with various RAD001 concentrations during a period of $72 \mathrm{~h}$.

According to our knowledge, this is the first report on the influence of RAD001 on chemically-induced urothelial tumorigenesis in mice. The dosage used in our study seemed to be well tolerated and no macroscopic changes were seen in the liver, lungs, kidneys, and gastrointestinal tract of the animals involved.

As determined by the observed results, the development of BBN induced urothelial lesions in mice was not significantly influenced by RAD001 treatments. Although there were few mice in each group, the incidence of urothelial preneoplastic lesions was slightly lower in those animals treated with RAD001 compared with animals exposed to BBN but not treated. The incidence of invasive lesions was not affected by RAD001 treatments. The rate of low-grade noninvasive papillary urothelial carcinoma was higher in 

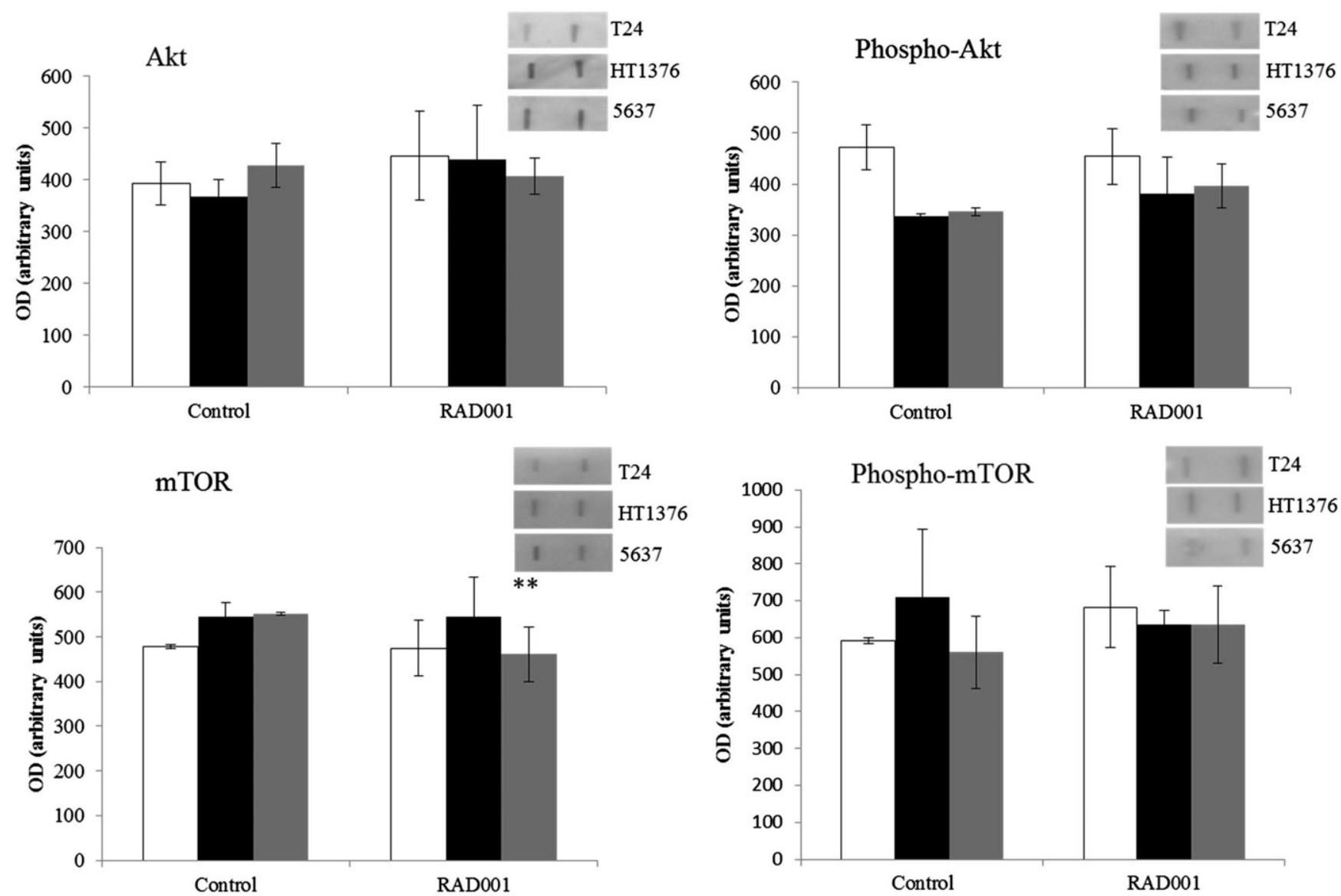

Fig. 6. Effect of $2 \mu \mathrm{M}$ RAD001 on the expression of Akt, phospho-Akt, mTOR and phospho-mTOR in bladder cell lines (T24 [white bars], HT1376 [black bars] and 5637 [grey bars]). Representative immunoblots are presented above the corresponding graphs. Data shown and bars represent the mean values \pm SD. $* * p<0.01$ versus untreated controls.

the group treated with RAD001. In our previous study using the same murine model (animals and time of exposition to BBN), sirolimus was more effective, significantly reducing the development of invasive lesions in comparison to nontreated mice [6].

In the in vitro study, heterogeneous results were observed. RAD001 had no effect on cellular proliferation and apoptosis of T24 and HT1376 cell lines considering the observed maintenance of Akt and mTOR expression and the light increase of this phosphorylated forms. However, a significant $\mathrm{S}$-phase decrease and a concomitant arrest of cells in $G_{0} / G_{1}$ cell cycle phase were seen in the 5637 cancer cell line, the only cell line studied in which a significant decrease of mTOR expression was observed. These effects were correlated to drug concentration and consistent with those observed in the MTT assay. With regard to apoptosis, a slight but significant increase of the sub- $\mathrm{G}_{0} / \mathrm{G}_{1}$ fraction was only observed in the 5637 cancer cell line. The RAD001 related reduction of mTOR observed on 5637 cell was associated with a slight increase of upstream phospho-Akt. Therefore, inhibiting mTORC1 release, the feedback inhibition mediated by the S6K-IRS1-PI3K loop that can lead to a paradoxical increase in Akt activity. Increased phosphorylated Akt has been detected in tumor biopsies from patients treated with rapalogs (mTOR inhibitors) [28].

Mansure et al. (2009) [27] found that RAD001 potently inhibits proliferation in some of several cell lines (UMUC-3, UM-UC-5, UM-UC-6, UM-UC-9, and UM-UC-14) with different stages of bladder tumorigenesis in vitro and also significantly inhibits human bladder KU-7 tumor xenografts growing in nude mice, though no concomitant apoptotic tumor cells were observed. These results revealed a cytostatic effect with no significant change in the sub- $G_{1}$ fraction and no cell loss in MTT assays.

Chiong et al. (2009) [29] also investigated the effect RAD001 had on human bladder-cancer cells (UM-UC3, UM-UC13) in vitro and in vivo. They observed heterogeneity in terms of in vitro responses to RAD001. The effect of RAD001 was augmented by retreatment of the cells after 3 days. After retreatment, only sensitive cell lines showed $\mathrm{G}_{1}$ phase arrest, with no evidence of apoptosis. However, RAD001 did significantly inhibit the growth of tumor cells that were subcutaneously implanted in mice. Both authors found that the anti-tumor activity of RAD001 is related to angiogenesis inhibition. This was evidenced by the decrease 
in VEGF production in vitro, and a significant reduction in microvessels.

Curiously, in a similar direction, we previously observed that 5637 cells, our most sensitive cell line, expressed nearly 4 times more VEGF-A than any other bladder cell lines studied [22]. Therefore, angiogenesis inhibition may be the predominant mechanism effect of sirolimus and its analogues [25,30].

It is important to underline that changes in tumor volume and angiogenesis observed in xenografts (nude mice) induced by RAD001 cannot be compared with the development of chemically induced bladder cancer in immunecompetent mice. More studies including the evaluation of protein synthesis inhibition through the S6K and 4E-BP1 pathways, in order to clarify RAD001's role in modulating the carcinogenesis process, are required using this murine model. In our opinion and according to our results, it is important to evaluate the effect of RAD001 prior to tumors being established, during the existence of pre-neoplastic lesions, which means before 12 weeks of $\mathrm{BBN}$ exposition.

In summary, RAD001 does not seem to have a significant effect on chemically induced murine bladder tumorigenesis. The treatment was well tolerated. The effect of RAD001 on proliferation and apoptosis across different bladder cancer cell lines is heterogeneous. Current knowledge suggests the possibility of rapalogs integration in bladder cancer treatment protocol. However, RAD001 needs more clarification regarding the mechanism of mTOR regulation and its role in the treatment of bladder cancer.

\section{Acknowledgments}

The authors express their deepest appreciation to Mrs. Lígia Lourenço for her technical assistance.

\section{References}

[1] Jemal A, Siegel R, Ward E, et al. Cancer Statistics, 2009. CA Cancer $\mathrm{J}$ Clin 2009;59:225-49.

[2] Winer E, Gralow J, Diller L, et al. Clinical Cancer Advances 2008: Major research advances in cancer treatment, prevention, and screening - a report from the American Society of Clinical Oncology. J Clin Oncol 2009;27:812-26.

[3] Stein JP, Skinner DG. Radical cystectomy for invasive bladder cancer: Long-term results of a standard procedure. World J Urol 2006; 3:296-304.

[4] Crallan RA, Georgopoulos NT, Southgate J. Experimental models of human bladder carcinogenesis. Carcinogenesis 2006;27:374-81.

[5] Oliveira PA, Colaço A, De la Cruz PL, et al. Experimental bladder carcinogenesis-rodent models. Exp Oncol 2006;28:2-11.

[6] Oliveira PA, Arantes-Rodrigues R, Sousa-Diniz C, et al. The effects of sirolimus on urothelial lesions chemically induced in ICR mice by BBN. Anticancer Res 2009;29:3221-6.

[7] Palmeira C, Lameiras C, Amaro T, et al. CIS is a surrogate marker of genetic instability and field carcinogenesis in the urothelial mucosa. Urol Oncol 2011;29:205-11.
[8] Ma WW, Adjei AA. Novel agents on the horizon for cancer therapy. CA Cancer J Clin 2009;59:111-37.

[9] Seghal SN. Sirolimus: Its discovery, biological properties, and mechanism of action. Transplant Proc 2003;35(3 Suppl):7S-14S.

[10] Edinger AL, Linardic CM, Chiang GG, et al. Differential effects of rapamycin on mammalian target of rapamycin signaling functions in mammalian Cells. Cancer Res 2003;63:8451-60.

[11] Paghdal K, Schwartz RA. Sirolimus (rapamycin): From the soil of Easter Island to a bright future. J Am Acad Dermatol 2007;57: 1046-50.

[12] Garcia J, Danielpour D. Mammalian target of rapamycin inhibition as a therapeutic strategy in the management of urologic malignancies. Mol Cancer Ther 2008;7:347-54.

[13] Boulay A, Zumstein-Mecker S, Stephan C, et al. Antitumor efficacy of intermittent treatment schedules with the rapamycin derivative RAD001 correlates with prolonged inactivation of ribosomal protein S6 kinase 1 in peripheral blood mononuclear cells. Cancer Res 2004; 64:252-61.

[14] Huang S, Bjornsti M, Houghton PJ. Rapamycins: Mechanism of action and cellular resistance. Cancer Biol Ther 2003;2:222-32.

[15] Sansal I, Sellers WR. The biology and clinical relevance of the PTEN tumor suppressor pathway. J Clin Oncol 2004;22:2954-63.

[16] Augustine JJ, Hricik DE. Experience with everolimus. Transplant P 2004;36(Suppl 2S):500S-503S.

[17] Kirchner GI, Meier-Wiedenbach I, Manns MP. Clinical pharmacokinetics of everolimus. Clin Pharmacokinet 2004;43:83-95.

[18] Janus A, Robak T, Smolewski P. The mammalian target of the rapamycin (mTOR) kinase pathway. Its role in tumorigenesis and targeted antitumor therapy. Cell Mol Biol Lett 2005;10:479-98.

[19] Koehl GE, Schlitt HJ, Geissler EK. Rapamycin and tumor growth: Mechanism behind its anticancer activity. Transplant R 2005;19: 20-31.

[20] Mabuchi S, Altomare DA, Cheung M, et al. RAD001 inhibits human ovarian cancer cell proliferation, enhances cisplatin-induced apoptosis, and prolongs survival in an ovarian cancer model. Clin Cancer Res 2007;13:4261-70.

[21] Epstein JI, Amim BM, Reuter VR, et al. World Health Organization/ International Society of Urological Pathology Consensus Classification of Urothelial (transitional cell) neoplasms of the urinary bladder. Am J Surg Pathol 1998;22:1435-48.

[22] Videira P, Piteira AR, Cabral MG, et al. Effects of bevacizumab on autocrine VEGF stimulation in bladder cancer cell lines. Urol Int 2001;86:95-101.

[23] Perabo FGE, Lindner H, Schmidt D, et al. Preclinical evaluation of gemcitabine/paclitaxel interactions in human bladder cancer lines. Anticancer Res 2003;23:4805-14.

[24] Pinto-Leite R, Botelho P, Ribeiro E, et al. Effect of sirolimus on urinary bladder cancer T24 cell line. J Exp Clin Can Res 2009;28:3.

[25] Fechner G, Claßen K, Schmidt D, et al. Rapamycin inhibits in vitro growth and release of angiogenetic factors in human bladder bancer. Urology 2009;73:665-8.

[26] Tang ZY, Wu YL, Gao SL, et al. Effects of the proteasome inhibitor bortezomib on gene expression profiles of pancreatic cancer cells. J Surg Res 2008;145:111-23.

[27] Mansure JJ, Nassim R, Chevalier S, et al. Inhibition of mammalian target of rapamycin as a therapeutic strategy in the management of bladder cancer. Cancer Biol Ther 2009;8:2339-47.

[28] Markman B, Dienstmann R, Tabernero J. Targeting the PI3K/Akt/ mTOR pathway - beyond rapalogs. Oncotarget 2010;1:530-43.

[29] Chiong E, Lee I-L, Dadbin A, et al. Effects of mTOR inhibitor everolimus (RAD001) on bladder cancer cells. Clin Cancer Res 2011;17:2863-73.

[30] Tickoo S, Satish K, Milowsky MI, et al. Hypoxia-inducible factor and mammalian target of rapamycin pathway markers in urothelial carcinoma of the bladder: Possible therapeutic implications. BJU Int 2011;107:844-9. 